\title{
ACTIVE-STERILE NEUTRINO OSCILLATIONS IN THE EARLY UNIVERSE AND THE ATMOSPHERIC NEUTRINO ANOMALY
}

\author{
P. DI BARI \\ Dipartimento di Fisica, Università di Roma 'La Sapienza' and INFN Roma 1, P.le \\ Aldo Moro, 2, I00185 Roma, Italy \\ E-mail: dibari@roma1.infn.it
}

\begin{abstract}
Cosmology cannot rule out the solution $\nu_{\mu} \leftrightarrow \nu_{s}$ to the atmospheric neutrino data and thus only Earth experiments will be able to give a definitive answer. This conclusion holds when a generation of lepton number is taken into account and one assumes that the sterile neutrino is also slightly mixed with an $\mathrm{eV}-\tau$ neutrino. This result cannot be spoiled by a chaotic generation of lepton domains.
\end{abstract}

\section{Severe BBN constraints in the two neutrino mixing scenario}

The atmospheric neutrino data are nicely explained in terms of neutrino oscillations $\nu_{\mu} \leftrightarrow \nu_{\alpha}$. Even though recent results favour the solution $\alpha=\tau$, the possibility that $\nu_{\alpha}$ is a sterile neutrino is still not completely excluded by Earth experiments. In anycase it is an interesting issue to know whether the BBN bound is able to rule out the solution $\nu_{\mu} \leftrightarrow \nu_{s}$. Neutrino oscillations are potentially able to thermalize the sterile neutrino that would thus contribute as a fourth neutrino species to the expansion rate, modifying the Standard BBN results. The recent indications from quasar absorbers for low values of Deuterium abundance $\left(D / H \sim 3.4 \times 10^{-5}\right)$ 1), suggest a BBN bound $\Delta N_{\nu}^{\mathrm{eff}}<0.9(0.6)$ at $99.7 \%(95.4 \%)$ c.l. . 百. In this case the solution $\nu_{\mu} \leftrightarrow \nu_{s}$ to the atmospheric neutrino data is definitely ruled out, as it is shown in the left

panel of figure 1. In this figure the constraints in the $\sin ^{2} 2 \theta_{0}-\left|\delta m^{2}\right|$ plane have been calculated analytically $\mathrm{B}$ in the effective kinetic approach (static approximation) developed by Foot and Volkas 4 国. They are in a good agreement with the numerical calculations 1 . Moreover they are valid when it is assumed that the effective total lepton number $L \equiv 2 L_{\nu_{\mu}}+L_{\nu_{\tau}}+L_{\nu_{e}}-(1 / 2) B_{n}$ starts and remains negligible. The quantities $Q_{X} \equiv\left(n_{X}-n_{\bar{X}}\right) / n_{\gamma}(Q=L$ or $B$ ) are the asymmetries of the particle species $X$. For positive $\delta m^{2}$ this picture is correct, but for negative ${ }^{a} \delta m^{2}$, even though one starts from a situation in which $L$ is initially negligible $\left(L \ll 10^{-6}\right)$, at a critical temperature $T_{c} \simeq 18 \mathrm{MeV}\left|\delta m^{2}\right|^{1 / 6}$ this can undergo a phase of rapid growth, first expo-

${ }^{a}$ We define $\delta m^{2} \equiv m_{2}^{2}-m_{1}^{2}$, with $m_{1}\left(m_{2}\right)$ the eigenvalue of the mass eigenstate coinciding with the active (sterile) neutrino interaction eigenstate for zero mixing. 

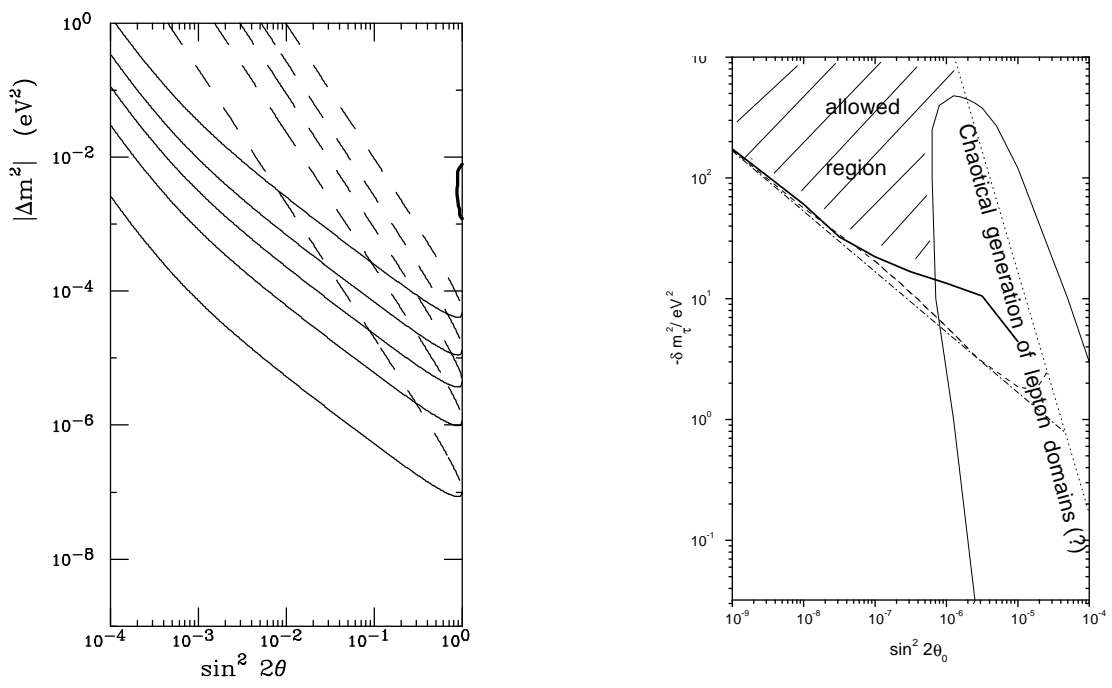

Figure 1. Left: constraints on the mixing parameters for $\nu_{\mu} \leftrightarrow \nu_{s}$. The dashed lines correspond the non resonant case positive $\delta m^{2}$ ), the thin solid lines to the resonant one (negative $\left.\delta m^{2}\right)$. From bottom up the different lines correspond to $N_{\nu_{s}}^{\text {eff }}=0.1,0.3,0.5,0.7,0.9$; the allowed region for the solution of the atmospheric neutrino problem is also shown (thick solid line). Right: allowed region in the three neutrino mixing mechanism.

nentially and afterwards as a power-law 缃. The presence of a large lepton number suppresses the sterile neutrino production $\mathrm{E}_{3}$ and thus it is legitimate to suspect whether taking into account the generation of lepton number can relax the constraints. The answer is negative. First, the growth occurs only for very small mixing angles and thus in anycase the BBN bound for the atmospheric neutrino solution cannot be evaded and moreover, even for small mixing angles, it occurs too late, when sterile neutrinos have already mostly been produced: therefore the constraints cannot be significantly relaxed. The account of lepton number generation is thus uneffective in a simple two neutrino mixing scenario.

\section{Three neutrino mixing mechanism to evade the BBN bound}

Assuming that the sterile neutrino is also slightly mixed with a heavier tau neutrino, a lepton number $L$ can be generated during $\nu_{\tau} \leftrightarrow \nu_{s}$ oscillations and it can afterwards suppress the sterile neutrino production during the $\nu_{\mu} \leftrightarrow \nu_{s}$ oscillations 0 . Neutrino oscillations are maximally active at $T_{c} \propto\left|\delta m^{2}\right|^{\frac{1}{6}}$. In 
this case we have two different $T_{c}$, associated with the two different $\delta m^{2}$ that we indicate with $\delta m_{\mu}^{2}$ and $\delta m_{\tau}^{2}$. Therefore, to have a generation of lepton number before the sterile neutrino production, one has to impose that $\delta m_{\tau}^{2}>$

$\delta m_{\mu}^{2}$. Moreover it is clear that one has also to impose that a significant sterile neutrino production does not occur already during the oscillations $\nu_{\tau} \leftrightarrow \nu_{s}$. Therefore the constraints discussed previously in the two neutrino mixing scenario must be imposed on the mixing parameters of tau neutrino (dotted line in right side of figure 1). These conditions are still not sufficient and things are made more complicate considering that the two different neutrino oscillations are mutually dependent. While the lepton number is produced from $\nu_{\tau} \leftrightarrow \nu_{s}$, it has also the effect of increasing the temperature $T_{c}$ for $\nu_{\mu} \leftrightarrow \nu_{s}$ oscillations that are anticipated and participate to the rate of growth of lepton number but with a destroying contribution. If this counter effect is dominant, first the lepton number stops its growth and then is completely destroyed. To avoid this situation the condition $\delta m_{\tau}^{2}>\delta m_{\mu}^{2}$ must be largely satisfied. However structure formation arguments do not allow a tau neutrino mass much higher than a few eV's and thus the lower limit on the $\delta m_{\tau}^{2}$ must not be much larger than about $10 \mathrm{eV}^{2}$. This can be determined analitically 10 in the effective kinetic approach. The rate of the total lepton number $L$ is simply given by $d L / d t=2 d L_{\nu_{\mu}} / d t+d L_{\nu_{\tau}} / d t$. The first term is the contribution from $\nu_{\mu} \leftrightarrow \nu_{s}$ and always destroys $|L|$, while the second is the contribution from $\nu_{\tau} \leftrightarrow \nu_{s}$ that at the critical temperature drive the growth of $|L|$. It is possible to show that $\left|d L_{\nu_{\alpha}} / d t\right|=k_{\alpha} \sin ^{2} \theta_{\alpha}\left|\delta m_{\alpha}^{2}\right|$ where $k_{\alpha}$ is a function of time and does not depend on the mixing parameters. The numerical analysis shows 3 that if the lepton number stops only once during its growth, then its fate is to be destroyed, otherwise it can grow up to a final value able to suppress the sterile neutrino production. In this way the condition that one has to impose for the lepton number to grow is simply that $d|L| / d t>0$ at any time. This is equivalent to impose the condition $\left|\delta m_{\tau}^{2}\right|>\sqrt{C}\left|\delta m_{\mu}^{2}\right| / \sqrt{s_{\tau}^{2}}$ where $C$ is the maximum of the ratio $k_{\mu} / k_{\tau}$ during the evolution of the lepton number. In the right panel of figure 1 the the dotdashed line corresponds to $\left|\delta m_{\mu}^{2}\right|=10^{-3} \mathrm{eV}^{2}$ and $C=28$, the value that gives the best fit of the numerical result obtained using the static approximation (dashed line). The thick solid line is the result of a numerical calculation in which the full quantum kinetic equations have been used $\mathbf{6}$.

\section{Chaotical generation of lepton domains?}

The three neutrino mixing mechanism is independent on the final sign of the lepton number. If however the final sign is sensitive to small fluctuations,

cosmo99: submitted to World Scientific on December 19, 2018 
one can imagine that different points of the early Universe eyglve a different sign and that a chaotical generation of lepton domains occurs 4 . In this case one should calculate a further sterile neutrino production deriving from those neutrinos that, crossing the boundaries of lepton domains, encounter a new resonance. This additive production could spoil the evasion of the BBN bound

9. Is a chaotical generation of lepton domains possible? A definitive answer to this difficult problem can be obtained only performing the full quantum kinetic calculations including momentum dependence 10 . The results show that the sign is fully determined for a large choice of mixing parameters and only in a restricted region the numerical analysis cannot be conclusive at the present. This region is the thin solid line in the right panel of the figure. It is evident that even assuming that a chaotical generation of lepton domains occurs in this region, determining a sterile neutrino overproduction, the allowed region for the three neutrino mixing mechanism still includes values of $\delta m^{2} \ll 100 \mathrm{eV}^{2}$, corresponding to a tau neutrino mass of a few eV's. Thus the conclusion is that cosmology cannot exclude the solution $\nu_{\mu} \leftrightarrow \nu_{s}$ to the atmospheric neutrino anomaly.

\section{Acknowledgments}

I wish to thank Robert Foot, Paolo Lipari, Maurizio Lusignoli and Ray Volkas for the collaboration and the encouragement during the period of thesis; A.D. Dolgov, K. Enqvist, K. Jedamzik, K. Kainulainen, S. Pastor and A. Sorri for nice discussions during the meeting; the organizers for a great conference.

\section{References}

1. D. Kirkman, D. Tytler, S. Burles, D. Lubin and J.M. O'Meara, astroph/9907128; S.A. Levshakov, astro-ph/0001406.

2. E. Lisi, S. Sarkar and F.L. Villante, Phys.Rev. D59 (1999) 123520.

3. P. Di Bari, P. Lipari, and M. Lusignoli, Int. J. Mod. Phys. A (in press), hep-ph/9907548.

4. R. Foot, M.J. Thomson and R.R. Volkas, Phys.Rev. D53, 5349 (1996).

5. R. Foot and R.R. Volkas, Phys.Rev. D55, 5147 (1997).

6. N.F. Bell, R.R. Volkas and Y.Y.Y. Wong, Phys.Rev. D59, 113001 (1999).

7. K. Enqvist, K. Kainulainen and M.J. Thomson, Nucl.Phys. B373, 498 (1992); X. Shi, D.N. Schramm and B.D. Fields, PR D48, 2568 (1993).

8. R. Foot, Astropart. Phys. 10, 253 (1999).

9. X. Shi and G. Fuller, Phys.Rev.Lett. 83, 3120 (1999).

10. P.Di Bari and R.Foot, accepted on Physical Review D, hep-ph/9912215.

cosmo99: submitted to World Scientific on December 19, 2018 